\title{
Head and Neck Survivorship Care in the Times of the SARS-CoV-2 Pandemic
}

\author{
Shaun A. Nguyen, MD, FAPCR ${ }^{1}$ \\ ${ }^{1}$ Medical University of South Carolina
}

April 27, 2020

\begin{abstract}
With the arrival of the coronavirus disease (SARS-CoV-2) in the U.S., care practice paradigms have drastically changed. Data from China suggests the new virus poses additional risks as case fatality of patients with cancer was higher at $5.6 \%$ compared to $2.3 \%$ of the general population. There are three proposed major strategies to address care for patients with cancer in this SARS-CoV-2 pandemic with postponing treatment for those with stable cancer, increasing personal protection provisions for cancer patients, and increasing monitoring if a patient becomes infected with SARS-CoV-2. In this present commentary, we discuss the unique mental health challenges and burdens of head and neck cancer (HNC) patients in the times of the SARSCoV-2 pandemic and approaches to mitigate these stressors through telemedicine to reduce future burdens to the patient and the health care system.

Victoria W. Huang, BA ${ }^{1}$, Sarah A. Imam, MD², Shaun A. Nguyen, MD $^{3}$

${ }^{1}$ Case Western Reserve University, Cleveland, OH, USA

${ }^{2}$ Department of Health and Human Performance, The Citadel, Charleston, SC, USA.

${ }^{3}$ Department of Otolaryngology - Head and Neck Surgery, Medical University of South Carolina, Charleston, $\mathrm{SC}, \mathrm{USA}$
\end{abstract}

Material has not been published previously.

None of the authors have any conflicts of interest, financial or otherwise.

No financial support.

All authors contributed substantially to, and approve of, this manuscript.

Corresponding Author:

Shaun A. Nguyen, MD, FAPCR

135 Rutledge Avenue, MSC 550

Charleston, SC 29425

Phone: (843) 792-1356

Email: Nguyensh@musc.edu

\section{Abstract}


With the arrival of the coronavirus disease (SARS-CoV-2) in the U.S., care practice paradigms have drastically changed. Data from China suggests the new virus poses additional risks as case fatality of patients with cancer was higher at $5.6 \%$ compared to $2.3 \%$ of the general population. There are three proposed major strategies to address care for patients with cancer in this SARS-CoV-2 pandemic with postponing treatment for those with stable cancer, increasing personal protection provisions for cancer patients, and increasing monitoring if a patient becomes infected with SARS-CoV-2. In this present commentary, we discuss the unique mental health challenges and burdens of head and neck cancer (HNC) patients in the times of the SARS-CoV-2 pandemic and approaches to mitigate these stressors through telemedicine to reduce future burdens to the patient and the health care system.

\section{Introduction}

With the arrival of the coronavirus disease (SARS-CoV-2) in the U.S., care practice paradigms have drastically changed. Social-distancing remains the most effective way to limit disease spread as the cases of SARS-CoV-2 continues to rise with no available vaccine or treatment ${ }^{1}$. This has resulted in the cancellation of multiple clinics and delay of procedures to limit the spread of the virus. For cancer patients who already have decreased immunity, data from China suggests the new virus poses additional risks as case fatality of patients with cancer was higher at $5.6 \%$ compared to $2.3 \%$ of the general population ${ }^{2}$. Another case study found that patients who had undergone anti-tumor therapy within 14 days of SARS-CoV-2 diagnosis had an increased risk of developing severe events and poorer outcomes ${ }^{3}$. Liang et al. proposed three major strategies to address care for patients with cancer in this SARS-CoV-2 pandemic with postponing treatment for those with stable cancer, increasing personal protection provisions for cancer patients, and increasing monitoring if a patient becomes infected with SARS-CoV-2 $2^{4}$.

In regards to postponing treatment, the National Comprehensive Cancer Network has released broad guidelines stating that for patients with solid tumors, adjuvant therapy with curative intent should proceed, despite the threat of SARS-CoV-2 infection during treatment ${ }^{5}$. The American College of Surgeons has also released guidance for the triage of non-emergent surgical procedures, recognizing that some elective cases intend to treat diseases that progress at variable, disease-specific rates and thus must proceed ${ }^{6}$. Otolaryngology specific safety guidelines have delineated high risk procedures as those working with exposed airway and mucosal surfaces that may generate aerosols ${ }^{7}$. Thus, prior to any surgical procedure, the SARS-CoV-2 status of a patient should be assessed with a discussion for delay of surgery if the patient is positive. With the current shortage of personal protective equipment and the need to reduce infection spread, institutions have developed processes to stratify the urgency of head and neck cases, delaying certain cases based on evidence from studies on prolonged time to treatment initiation. These treatment decisions aim to balance the unknown risk of infection to the patient, exposure of the health care workers and use of valuable personal protective equipment with the progression of cancer that may increase in mortality and likelihood of recurrence with delay. Studies have noted a poorer overall survival is associated with an extended period between diagnosis to initiation of treatment with delay thresholds ranging from 20 to 120 days ${ }^{8}$. Psychiatric vulnerabilities in certain cancer patients may also be exposed with extended wait times ${ }^{9}$. However, with the realities of the lack of personal protective equipment and the need to redirected staff to SARS-CoV-2 patients, there is a move toward delaying non-urgent cases. With the uncertainties of the SARS-CoV-2 pandemic and a head and neck cancer (HNC) diagnosis, the potential mental health consequences of such delays to treatment warrant further discussion.

HNC patients suffer from unique challenges, as much of social functioning depends on the structural and functional integrity of the head and neck region. Disease process and treatment can significantly alter this. Psychological distress is also particularly prevalent in HNC patients as nearly $35 \%$ of patients suffer from symptoms of depression and anxiety ${ }^{10}$. Now, with the added complications of the SARS-CoV-2 pandemic, restrictions on movement may increase patients' stress, depression, and fear ${ }^{11}$. In this present commentary, we discuss the unique mental health challenges and burdens of HNC patients in the times of the SARS-CoV-2 pandemic and approaches to mitigate these stressors through telemedicine to reduce future burdens to the 
patient and the health care system.

\section{Methods}

\section{Literature Review and Evidence Collection}

A literature review was performed by two authors (VWH, SAI) searching for all published English-language literature reporting on head and neck cancer in the SARS-CoV-2 era. A search strategy was employed with the following search strings: "virus OR corona OR covid OR survival" with "head and neck cancer" in the last 5 years on PubMed, Scopus, Cochrane Library, OVID, and Google. An independent search query was also employed with "head and neck mental health" and "head and neck survivorship". To identify additional articles, the reference lists of relevant articles were hand searched, as well as citing articles. Results were limited to English articles. After the completed search, a total list of records was obtained and duplicates removed. Articles were considered for discussion if they provided relevant quality of life measures and protocols for head and neck cancer care in the context of this SARS-CoV-2 pandemic. A final list of full text articles was then compiled and each article was then screened by one author.

\section{Discussion}

Once a patient is diagnosed with HNC, they enter into the continuum of cancer survivorship. A patient is deemed a cancer survivor from the moment of diagnosis. This terminology encourages providers and patients to consider the impact of diagnosis and treatment earlier as multiple studies have highlighted the unique experience of HNC patients as they progress through changes in swallowing, speech, and nutrition ${ }^{12}$. These factors have been studied as a part of quality of life measures which highlight the importance of not only cancer survival, but also a patient's experience of life after diagnosis. It is critical to consider these factors in the context of the SARS-CoV-2 pandemic.

HNC patients have reported feelings of isolation with a lack of social support following diagnosis ${ }^{13}$. The high morbidity and mortality associated with $\mathrm{HNC}$ along with stigma associated with the facial disfigurement following treatment can result in decreased quality of life (QOL) that may affect survival ${ }^{14}$. HNC patients at three Veterans' Affairs Hospitals reported QOL scores in pain, eating, and speech domains which were associated with survival ${ }^{15}$. A prospective study on HNC patients found QOL scores were associated with short-term, moderate, and long-term survival. Long-term survivors demonstrated the best QOL trajectory at 12 months following diagnosis, specifically in the domains of eating and speech ${ }^{16}$. Both studies showed no association of emotional scores with overall survival. However, there has been evidence that mental adjustment responses with either a "fighting spirit" versus hopelessness is an important prognostic factor in determining both death and recurrence ${ }^{17}$. The association between QOL and survival remains complex and requires continued exploration as cancer survivor numbers continue to increase ${ }^{18}$.

In addition to patient experience, the role of cancer specific characteristics and the patient's biological biomarkers have been investigated for its relation to QOL and mental health. For patients with human papillomavirus (HPV)-positive oral cavity and oropharyngeal cancers, there was a larger decrease in QOL from pretreatment to immediate posttreatment, suggesting that treatment intensity compared to non-HPV cancers may negatively affect QOL trajectories ${ }^{19}$. This significant decrease in QOL may also make HPVpositive patients more susceptible to mood disorders. An investigation into mental health insurance claims found that HNC patients had a higher prevalence of mental health diseases at $20.6 \%$, higher than the national estimate at $17.9 \%$. Following HNC diagnosis, the prevalence increased to $29.9 \%{ }^{20}$. A separate study highlighted that the prevalence of a functional genetic polymorphism of the serotonin transporter gene was not significantly different between control depression patients and HNC depression patients who had completed treatment ${ }^{21}$. Of note, $19 \%$ of patients developed depression after undergoing concurrent chemoradiation, suggesting a strong interaction of treatment with depression. However, depression may arise at any time during the cancer survivorship continuum. For HNC patients in the United Kingdom, the pretreatment phase was noted to be a time of high anxiety and depression that was associated with a poor $\mathrm{QOL}^{22}$. 
The burden of HNC diagnoses can extend beyond a mental challenge for patients. Caring for HNC patients can be a grand task as caregivers help patients navigate the complex follow-up appointments and changes in abilities to communicate and eat. HNC treatment can also result in poor outcomes for the caregiver, highlighting a need for caregiver interventions ${ }^{23}$. An additional financial burden is also placed on the patient and caregivers as Massa et. al noted a higher median annual medical expense and relative out-of-pocket expenses for patients with HNCs when compared to other cancers. This higher financial burden is placed upon HNC survivors who are already more often members of a minority race/ethnicity, poor, and less educated with lower general and mental health status ${ }^{24}$.

With HNC patients already a vulnerable population, delaying treatment in the times of the SARS-CoV-2 pandemic can place additional strain on the mental health and quality of life of patients, resulting in future burdens on the health care system. It is critical to help patients maintain their quality of life through this pandemic as it may affect their overall outcomes of survival. Recognizing the psychological toll social isolation may place on the population, China provided multiple telemental health services that encompassed virtual counseling, training, and psychoeducation ${ }^{25}$. Online behavioral therapy for depression, anxiety, and insomnia along with multiple books with guidelines for public psychological self-help and counselling were accessible during the times of the SARS-CoV-2 pandemic ${ }^{26}$. In the U.S., the CMS has loosened telehealth guidelines, increasing the platforms on which physicians may communicate with patients, while the AAOHNS has called for the adoption of telemedicine to decrease unnecessary exposure to both the patient and physician $^{27,28}$. To improve survivorship experience, we encourage the use of such resources to address the cancer patient's quality of life while institutions continue to triaging urgent surgeries to balance the risk of infection to HNC patients.

\section{Conclusion}

As the SARS-CoV-2 pandemic continues and social distancing remains common practice, the mental health toll and effects on quality of life for HNC patients need to be anticipated. HNC patients carry a larger burden of financial costs and potential for mental health deterioration that may affect ultimate outcomes. With examples of utilizing technology from China, providers need to address the mental health burden of a head and neck cancer diagnosis for patients who are being asked to delay treatment to ensure comprehensive cancer care.

\section{Bibliography}

1. Lewnard JA, Lo NC. Comment Scientific and ethical basis for social-distancing interventions against COVID-19. Lancet Infect Dis . 2020;3099(20):1-2. doi:10.1016/S1473-3099(20)30190-0

2. Wu Z, McGoogan JM. Characteristics of and Important Lessons From the Coronavirus Disease 2019 ( COVID-19 ) Outbreak in China Summary of a Report of 72314 Cases From the Chinese Center for Disease Control and Prevention. JAMA . 2020;323(13):1239-1242.

3. Zhang L, Zhu F, Xie L, et al. Clinical characteristics of COVID-19-infected cancer patients: A retrospective case study in three hospitals within Wuhan, China. Ann Oncol . 2020. doi:10.1016/j.annonc.2020.03.296

4. Liang W, Guan W, Chen R, Wang W. Cancer patients in SARS-CoV-2 infection : a nationwide analysis in China. Lancet . 2020;2045(20):335-337. doi:10.1016/S1470-2045(20)30096-6

5. Ueda M, Martins R, Hendrie PC, et al. Managing Cancer Care During the COVID-19 Pandemic: Agility and Collaboration Toward a Common Goal.J Natl Compr Cancer Netw . 2020;18(4):366-369. doi: $10.6004 /$ jnccn.2020.7560

6. American College of Surgeons. COVID-19: Guidance for Triage of Non-Emergent Surgical Procedures.

7. Givi B, Schiff B, Chinn SB, et al. Safety Recommendations for Evaluation and Surgery of the Head and Neck During the COVID-19 Pandemic. JAMA Otolaryngol Neck Surg . 2020:e1-6. doi:10.1001/jamaoto.2020.0780 
8. Graboyes EM, Kompelli AR, Neskey DM, et al. Association of Treatment Delays With Survival for Patients With Head and Neck Cancer: A Systematic Review. JAMA Otolaryngol Neck Surg . 2019;145(2):166177. doi:10.1001/jamaoto.2018.2716

9. Song H, Fang F, Valdimarsdóttir U, et al. Waiting time for cancer treatment and mental health among patients with newly diagnosed esophageal or gastric cancer : a nationwide cohort study. BMC Cancer . 2017;17(2):1-9. doi:10.1186/s12885-016-3013-7

10. Riblet N, Skalla K, Mcclure A, Homa K, Luciano A, Davis TH. Addressing Distress in Patients With Head and Neck Cancers: A Mental Health Quality Improvement Project. J Natl Compr Cancer Netw . 2014;12(7):1005-1013.

11. Pfefferbaum B, North CS. Mental Health and the Covid-19 Pandemic.N Engl J Med . 2020:1-3.

12. Pollack LA, Greer GE, Rowland JH, et al. Cancer survivorship a new challenge in comprehensive cancer control. Cancer Causes Control . 2005;16:51-59. doi:10.1007/s10552-005-0452-x

13. D'Antonio LL, Long SA, Zimmerman GJ, Peterman AH, Petti GH, Chonkich GD. Relationship Between Quality of Life and Depression in Patients With Head and Neck Cancer. Laryngoscope . 1998;108(June):806811.

14. Callahan C. Facial Disfigurement and Sense of Self in Head and Neck Cancer. Soc Work Health Care . 2005;40(2):73-87. doi:10.1300/J010v40n02

15. Karvonen-gutierrez CA, Ronis DL, Fowler KE, Terrell JE, Gruber SB, Duffy SA. Quality of Life Scores Predict Survival Among Patients With Head and Neck Cancer. J Clin Oncol . 2008;26(16):2754-2760. doi:10.1200/JCO.2007.12.9510

16. Goldstein DP, Karnell LH, Christensen AJ, Funk GF. Health-related quality of life profiles based on survivorship status for head and neck cancer patients. Head Neck . 2007;(March):221-229. doi:10.1002/hed

17. Pettingale KW, Morris T, Greer S, Haybittle JL. Mental Attitudes to Cancer: An Additional Prognostic Factor. Lancet . 1985;1(8431):750.

18. Verdonck-de Leeuw I, Jansen F, Brakenhoff RH, Langendijk JA, Takes R, Terhaard CHJ. Advancing interdisciplinary research in head and neck cancer through a multicenter longitudinal prospective cohort study : the NETherlands QUality of life and BIomedical Cohort ( NET-QUBIC ) data warehouse and biobank. BMC Cancer. 2019;19(765):1-13.

19. Sharma A, Mendez E, Yueh B, et al. Human Papillomavirus-Positive Oral Cavity and Oropharyngeal Cancer Patients Do Not Have Better Quality-of-Life Trajectories. Otolaryngol Neck Surg . 2012;146(5):739745. doi:10.1177/0194599811434707

20. Lee JH, Ba D, Liu G, Leslie D, Zacharia BE, Goyal N. Association of Head and Neck Cancer With Mental Health Disorders in a Large Insurance Claims Database. JAMA Otolaryngol Neck Surg . 2019;145(4):339344. doi:10.1001/jamaoto.2018.4512

21. Gilbert J, Haman KL, Dietrich MS, Blakely RD, Shelton RC, Murphy BA. Depression in patients with head and enck cancer and a functional genetic polymorphism of the serotonin transporter gene. Head Neck . 2012;(March):359-364. doi:10.1002/hed

22. Horney DJ, Smith HE, Mcgurk M, et al. Associations between quality of life, coping styles, optimism, and anxiety and depression in pretreatment patients with head and neck cancer. Head Neck . 2011;(January):6571. doi:10.1002/hed

23. Sterba KR, Zapka J, Armeson KE, et al. Physical and emotional well-being and support in newly diagnosed head and neck cancer patient-caregiver dyads. J Psychosoc Oncol . 2018;35(6):646-665. doi:10.1080/07347332.2017.132 
24. Massa ST, Osazuwa-peters N, Boakye EA, Walker RJ, Ward GM. Comparison of the Financial Burden of Survivors of Head and Neck Cancer With Other Cancer Survivors. JAMA Otolaryngol Neck Surg . 2019;145(3):239-249. doi:10.1001/jamaoto.2018.3982

25. Zhou X, Snoswell MMHCL, Harding LE, Bambling M, Edirippulige S, Bai X. The Role of Telehealth in Reducing the Mental Health Burden from COVID-19. Telemed e-Health . 2020;26(4):26-28. doi:10.1089/tmj.2020.0068

26. Liu S, Yang L, Zhang C, et al. Correspondence Online mental health services in China during the COVID-19 outbreak. The Lancet Psychiatry . 2020;7(4):e17-e18. doi:10.1016/S2215-0366(20)30077-8

27. Centers for Medicare and Medicaid Services. Medicare Telemedicine Health Care Provider Fact Sheet. https://www.cms.gov/newsroom/fact-sheets/medicare-telemedicine-health-care-provider-fact-sheet. Accessed March 24, 2020.

28. American Academy of Otolaryngology - Head and Neck Surgery. Prioritizing Novel Approaches to Telehealth for All Practitioners. https://www.entnet.org/content/prioritizing-novel-approaches-telehealthall-practitioners. Accessed March 24, 2020. 